\title{
Quantum Field Model of the Origin of Matter-Energy in the Expanding Universe
}

\author{
V.E. Kuzmichev, V.V. Kuzmichev* \\ Bogolyubov Institute for Theoretical Physics, National Academy of Sciences of Ukraine, Kiev, 03680 Ukraine
}

*Corresponding Author: specrada@bitp.kiev.ua

Copyright (C)2013 Horizon Research Publishing All rights reserved.

\begin{abstract}
The model of production of ordinary and dark matter in the decay of a hypothetical antigravitating medium in the form of a condensate of (zero-momentum) spinless massive particles, which fills the early universe, is proposed. The decays of these massive particles into baryons, leptons, and dark matter particles are caused by some (after-GUT) interaction with the mass scale between the electroweak and grand unification. The observed dark energy is identified with a portion of a condensate which has not decayed up to the instant of measurement. We show that the mass of dark matter particle being close to $5 \mathrm{GeV}$ and the mass of massive particle of a condensate approximately equal to $15 \mathrm{GeV}$ can be extracted from the WMAP and other astrophysical data about the contributions of baryon, dark matter, and dark energy densities to the total matter-energy density budget in our universe. Such a mass of light WIMP dark matter agrees with the observations of CoGeNT, DAMA, and CDMS. The numerical values of the coupling constant of after-GUT interaction and of the decay rate of massive particle of a condensate, as well as other parameters are obtained.
\end{abstract}

Keywords Dark Energy, Dark Matter, Production of Baryons, Decay Rate

\section{Introduction}

The present data of modern cosmology pose the principle question about the origin and nature of massenergy constituents of our universe. The 9-year WMAP and other data [1] indicate that: the spatial geometry of the observed part of our universe is very close to flat; the expansion of the universe is accelerating due to the action of dominating substance called dark energy; the universe is now comprised of one third dark matter, which provides the formation of observed structures composed of baryons and leptons, and two thirds dark energy. It is remarkable that observed mass of stars is negligibly small (it accounts for $\approx 0.5 \%$ from the total amount of mass-energy in the universe $[1,2,3])$. In view of the current dominance of dark energy over all other forms of matter it is reasonable to consider a model in which ordinary and dark matter be decay products of a portion of some primordial antigravitating medium, which fills the universe, under the action of some interaction with gauge coupling between the electroweak and grand unification scales. This interaction leads to the violation of CP-invariance which can cause the baryon asymmetry of the universe. This process is characterized by a very small decay rate and the undecayed part of antigravitating medium can be considered as dark energy. The antigravitating medium itself can be identified with a scalar field called quintessence or can be related to the inflaton field which is used in inflation models [4].

This paper is written within the context of research directed towards the elimination of confrontation between observed value of the vacuum energy density $\rho_{\text {vac }} \sim 0.7 \rho_{\text {today }}$ (cosmological constant) [1] and its value $\rho_{\text {vac }}>10^{30} \rho_{\text {today }}{ }^{1}$ predicted by the quantum field theory $[5,6]$. The main idea is that the vacuum energy density has decreased during the expansion of the universe. Such a decrease can be explained by the decay of the corresponding vacuum state. Under such an approach, both the vacuum state itself and the mechanism of its decay are modeled (for details, see $[7,8]$ ). For example, in [7] the decay of symmetric vacuum state as evaporation of a Bose condensate of physical Higgs particles is studied.

In this paper the decaying antigravitating medium has a form of a Bose condensate of quantized primordial scalar field [9]. Starting with the Einstein-Hilbert action of general relativity with matter, the formalism is applied for the case of the homogeneous, isotropic and closed universe. The scalar field serves as a surrogate for the matter content of the universe. In order to pass from classical to quantum description, the Dirac's canonical quantization method [10], in which the constraints become operators that annihilate physical state vectors, is used and the notion of the so-called material reference system (related to relativistic elastic media introduced

\footnotetext{
${ }^{1} \mathrm{~A}$ particular value of $\rho_{v a c}$ depends on the value of the wavenumber cutoff which is a free parameter.
} 
by DeWitt $[11,12])$ is employed. After quantization the scalar field changes into a condensate whose properties are described by the quantum theory. The direct calculations show that, for quantum states with the mass of a condensate which exceeds significantly the Planck mass, a condensate acquires the properties of an antigravitating medium with the vacuum-type equation of state. If one discards the quantum effects, a condensate turns into an aggregate of separate macroscopic bodies with zero pressure which is known as dust and used to model ordinary matter in general relativity. The existence of this limit argues, first of all, in favour of reliability of the quantum model proposed in [9] and, secondly, exhibits the quantum nature of antigravitating property of dark energy.

In this paper we consider the nonstationary universe in which a condensate of a primordial scalar field is a source of matter in the form of baryons, leptons, and dark matter, while observed dark energy is a portion of a condensate which has not decayed up to the instant of observation. In Section 2 the decay rate $\Gamma_{\phi}$ of particle of a condensate ( $\phi$-particle) is derived under assumption of the existence of a new force mediated by new virtual massive $X$-particles with the coupling at the mass scale $M_{X}$ between the electroweak $M_{W}$ and grand unification $M_{G}$. In Section 3 the numerical values of unknown parameters, such as the mean decay rate $\bar{\Gamma}_{\phi}$, masses of $\phi$-particle and dark matter particle, are calculated. In Section 4 these values are used to obtain the numerical estimation for the mass scale $M_{X}$ and corresponding coupling constant $\alpha_{X}$. The estimations for the density of $\phi$-particles surrounded by virtual $X$-particle cloud and for the cross-section of the reactions in which dark matter particles can be produced are given. In Section 5 the conclusion remarks are drawn.

\section{Matter Production}

According to modern point of view (see, e.g., [13]), the very early universe (close to the Planck era) was filled with the relativistic matter with the kinetic energy of motion of its constituent particles (temperature $T$ ) which exceeds significantly their rest mass energy, $T \gg m_{\max }$, where $m_{\max }$ is the greatest mass of all the particles. Particles may acquire the mass after the spontaneous symmetry breaking under the cooling of the expanding universe. When the temperature falls to the value $T \ll m_{\min }$, where $m_{\text {min }}$ is the smallest mass of the particles, matter in the universe is found to be composed of two components, radiation and massive particles. In the early universe the relativistic matter itself may arise as a result of transition of the vacuum energy of some primordial scalar field into the energy of small quantum oscillations near the equilibrium state corresponding to true or false vacuum.

When applying this model to the study of the evolution of matter in the universe, it is convenient to represent the relativistic matter in the form of the sum of two components, one of which is composed of massless particles, while another consists of massive particles. Massless particles form the radiation with which the reference frame can be associated [9]. In the era $T \ll m_{m i n}$, massive particles produce ordinary and dark matter observed in the universe.

Following [9], massive component is considered as a condensate of scalar field quanta ( $\phi$-particles). Their mass $m_{\phi}$ can be expressed through the curvature of the potential energy density of a primordial scalar field. Stationary states of a condensate are characterized by a mass (energy), $M_{k}=m_{\phi}\left(k+\frac{1}{2}\right)$, where $M_{k}$ is the eigenvalue of the operator of mass-energy of a scalar field in a comoving volume, $k$ is the number of $\phi$-particles which are bosons. A condensate is a chargeless medium (for all types of charges). Therefore, $\phi$-particle coincides with its antiparticle, $\phi=\bar{\phi}$. The momentum of $\phi$-particle vanishes and its spin is $s_{\phi}=0$.

Hydrogen is the most abundant element in the observable universe. One can assume that its constituent elements (proton and electron) are formed in the early universe as a result of the decay of a neutron, which in turn is a decay product of some primordial matter. Let us choose a condensate of $\phi$-particles as a primordial matter and suppose that baryons $(n, p)$, leptons $\left(e^{-}, \nu_{e}\right)$, dark matter particle $(\chi)$ and their antiparticles $\left(\bar{n}, \bar{p}, e^{+}, \bar{\nu}_{e}, \bar{\chi}\right)$ are produced via the decays of $\phi-$ particles in the processes

$$
\phi \rightarrow \chi+\nu+n \text { and then } n \rightarrow p+e^{-}+\bar{\nu},
$$

or

$$
\phi=\bar{\phi} \rightarrow \bar{\chi}+\bar{\nu}+\bar{n} \text { and then } \quad \bar{n} \rightarrow \bar{p}+e^{+}+\nu .
$$

Within the framework of the quark model, the condensate under consideration can be a chargeless aggregate of point-like quarks and gluons, a kind of quark-gluon plasma. Then $\phi$-particles will be the particle-like excitations of this plasma. The particle production in the decays (1) and (2) can be considered as a process of evaporation of quarks bound together in the energyfavorable states in the form $\chi$ and $n$ particles. Neutrinos and antineutrinos ensure the law of angular momentum conservation. This brings us to the field of QCD and requires the new model assumptions about the mechanism of evaporation of particles. Therefore, for clarity, in order to focus on the final stage of the origin of matterenergy in the expanding universe, we shall consider a simplified phenomenological model in which quark degrees of freedom are not taken into account.

In every separate decay (1) or (2) the baryon invariance is violated. If both decays (1) and (2) are equiprobable, then the system composed from matter, antimatter (including dark sectors), and a fraction of $\phi$-particles of a condensate which have not decayed up to the instant under consideration, remains chargeless. The processes (1) and (2) imply that the dark matter particle and antiparticle are scalar $\left(\operatorname{spin} s_{\chi}=0\right)$ or vector $\left(s_{\chi}=1\right)$ particles. It follows from [14] that a dark matter particle may be a scalar particle with scalar interaction. The presence of the neutrino in the decay (1) guarantees the spin conservation law.

Particles and antiparticles in the decay products of the processes (1) and (2) can annihilate between themselves and contribute to the cosmic background radiation. Net amounts of protons, leptons, and dark matter particles constitute matter in the luminous and dark forms in the universe. 
Taking into account above-mentioned issues, let us examine the decay (1) in which dark and baryonic matter are produced. We consider this process using an analogy with neutron and proton decays. In the standard model, the decay of neutron is the result of weak interaction, mediated by virtual $W$-boson exchange,

$$
n \rightarrow p+W^{-} \text {and then } W^{-} \rightarrow e^{-}+\bar{\nu} .
$$

The rate $\Gamma_{n}$ of decay of a neutron into the final $p e \nu$-state is equal to

$$
\Gamma_{n}=\frac{1}{\tau_{n}}=\alpha_{W}^{2} \frac{\Delta m^{5}}{M_{W}^{4}}
$$

(in units $\hbar=c=1$ ), where $\tau_{n}$ is the mean life of neutron, $\Delta m=m_{n}-m_{p}$ is the energy imparted by the $W$-boson to the leptons $e \nu, M_{W}$ is the mass of the $W$ boson, $m_{n}$ and $m_{p}$ are the masses of neutron and proton, respectively. The quantity $\alpha_{W}^{2}$ is dimensionless constant which characterises the strength of weak interaction and includes small radiative and other quantum corrections. This number is close to the value $G_{F} m_{p}^{2}=1.027 \times 10^{-5}$, where $G_{F}=1.166 \times 10^{-5} \mathrm{GeV}^{-2}$ is the Fermi coupling constant,

$$
\alpha_{W}^{2}=G_{F} m_{p}^{2}(1-\delta) \sim 10^{-5},
$$

where $\delta \sim O\left(10^{-1}\right)$ is small correction.

In any grand unified theory (GUT) (see, e.g., the review [15]) the proton lifetime $\tau_{p}$ is given by the following relation

$$
\Gamma_{p}=\frac{1}{\tau_{p}}=\alpha_{G}^{2} \frac{m_{p}^{5}}{M_{G}^{4}},
$$

where $\Gamma_{p}$ is the decay rate of proton, $\alpha_{G}$ and $M_{G}$ are two parameters. Universal gauge coupling $\alpha_{G}$ is defined at the grand unification scale $M_{G}$. The SUSY GUTs give the value $\alpha_{G} \sim \frac{1}{25}$ at $M_{G} \sim 3 \times 10^{16} \mathrm{GeV}$. These parameters correspond to the proton lifetime equal to $\tau_{p} \sim 10^{37}$ yrs. We can use the estimation

$$
\alpha_{G}^{2} \sim 10^{-3}
$$

It can be compared with $\alpha_{W}^{2}$ from (5)

$$
\alpha_{W}^{2} \sim 10^{-5}<\alpha_{G}^{2} \sim 10^{-3} .
$$

Let us suppose that the decay of $\phi$-particle in the process (1) is mediated by exchange of virtual $X$-particles which are quanta of some new field with the mass scale

$$
M_{X} \gg m_{\chi}>m_{n} \gg m_{\nu}
$$

where $m_{\chi}$ and $m_{\nu}$ are the masses of dark matter particle and neutrino. Generally speaking, a few versions of such a decay are possible

$$
\begin{array}{lll}
\phi \rightarrow \chi+X & \text { and then } & X \rightarrow n+\nu, \\
\phi \rightarrow n+X & \text { and then } & X \rightarrow \chi+\nu \\
\phi \rightarrow \nu+X & \text { and then } & X \rightarrow \chi+n
\end{array}
$$

In Fig. 1, the diagram represents the first process in (10). The two other diagrams can be obtained by cyclic permutation of the particles $\chi, n, \nu$.

Since the spin $s_{\phi}=0$, one concludes that in the first process in (10) the spins of $\chi$ - and $X$-particles can vanish, $s_{\chi}=0$ and $s_{X}=0$, or be equal to unity, $s_{\chi}=1$ and

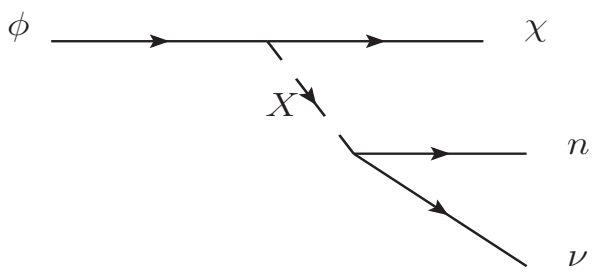

Figure 1. The diagram which corresponds to the first decay in (10).

$s_{X}=1$. In this case $\chi$ - and $X$-particles are scalars or vector bosons. In the second and third processes in (10) $\chi$-particle is still a scalar or vector boson, but $X$-particle is a fermion with the spin $s_{X}=\frac{1}{2}$.

We consider the processes (10) by analogy to those described by (4) and (6). We neglect the contribution from integration with respect to intermediate momentum of $X$-particle in corresponding transition amplitudes, as well as contributions into the decay rate of $\phi$-particle $\Gamma_{\phi}$ arising from all higher-order diagrams. Then $\Gamma_{\phi}$ can be written as follows

$$
\Gamma_{\phi}=\alpha_{X}^{2} \frac{Q^{5}}{M_{X}^{4}},
$$

where

$$
Q=m_{\phi}-\left(m_{\chi}+m_{n}+m_{\nu}\right)
$$

is the energy imparted by $\phi$-particle at rest to the decay products in (1). The dimensionless coupling constant $\alpha_{X}^{2}$ takes into account the contributions into $\Gamma_{\phi}$ from the first or both the second and third pole diagrams for the processes (10). In the case, when $X$-boson has a supersymmetric partner with the same mass ( $X$-fermion), all three pole diagrams contribute into the decay rate $\Gamma_{\phi}$.

Using (4), (6), and (11) one can write the expressions for the ratios of the decay rate of $\phi$-particle to the neutron and proton decay rates

$$
\frac{\Gamma_{\phi}}{\Gamma_{n}}=\frac{\alpha_{X}^{2}}{\alpha_{W}^{2}}\left(\frac{Q}{\Delta m}\right)^{5}\left(\frac{M_{W}}{M_{X}}\right)^{4}
$$

and

$$
\frac{\Gamma_{\phi}}{\Gamma_{p}}=\frac{\alpha_{X}^{2}}{\alpha_{G}^{2}}\left(\frac{Q}{m_{p}}\right)^{5}\left(\frac{M_{G}}{M_{X}}\right)^{4} .
$$

Here the quantities $\Gamma_{\phi}, \alpha_{X}^{2}, Q$ and $M_{X}$ are unknown. The decay rate $\Gamma_{\phi}$ and the parameter $Q$ can be calculated independently in the kinetic theory which considers two-step processes (1) and (2) as dynamical ones and uses the astrophysical data about the contributions of baryon, dark matter, and dark energy densities to the total matter-energy density budget in our universe.

\section{Dark Energy and Light WIMP Dark Matter}

The total energy density of matter in the universe relative to critical density $\Omega_{t o t}$ taken at some fixed instant of the proper time $t$ can be represented by the sum of the terms

$$
\Omega_{t o t}=\Omega_{B}+\Omega_{L}+\Omega_{D M}+\Omega_{C M B}+\Omega_{D E},
$$


where $\Omega_{B}, \Omega_{L}, \Omega_{D M}$, and $\Omega_{C M B}$ are the energy densities of baryons, leptons, dark matter particles, and the cosmic microwave background radiation, $\Omega_{D E}$ is the density of dark energy (i.e. a portion of a condensate which has not decayed up to the instant under consideration). These constituents can be written as follows

$$
\begin{array}{r}
\Omega_{B}=\frac{2 m_{p} \delta N_{p}(t)}{a^{3}(t) H^{2}(t)}, \quad \Omega_{L}=\frac{2 m_{l} \delta N_{l}(t)}{a^{3}(t) H^{2}(t)}, \\
\Omega_{D M}=\frac{2 m_{\chi} \delta N_{\chi}(t)}{a^{3}(t) H^{2}(t)}, \quad \Omega_{D E}=\frac{2 m_{\phi} \delta N_{\phi}(t)}{a^{3}(t) H^{2}(t)},
\end{array}
$$

where $a$ is the cosmic scale factor taken in unit of the Planck length $l_{P}=\sqrt{\frac{2 G}{3 \pi}}, m_{l}$ is the sum of masses of all leptons in the final state of reaction (1), and $H$ is the dimensionless Hubble expansion rate (in unit of time $\left.t_{P}=l_{P}\right)^{2}$. All masses in (16) are taken in units of the Planck mass $m_{P}=l_{P}^{-1}$. The quantities

$$
\delta N_{i}=N_{i}-N_{\bar{i}}, \quad i=\{p, l, \chi\},
$$

are the differences between the number of particles $N_{i}$ and antiparticles $N_{\bar{i}}$ of $i$ th type as the functions of $t$, $m_{i} \delta N_{i}$ is the total mass of particles of $i$ th type that remained after annihilation, $\delta N_{\phi}$ is the number of undecayed $\phi$-particles which form dark energy.

According to the quantum model description [9], when the universe expands, the following condition is realized at every instant of time for large enough number of the $\phi$-particles,

$$
\langle a\rangle_{k}=M_{k},
$$

where $\langle a\rangle_{k}$ is the mean value of the scale factor $a$ in the k-state of the universe with the mass of a condensate $M_{k}$ taken in Planck units. The equation similar to (18) was proposed by Sciama $[16,17]$ as an example of a mathematical formulation of the Mach's principle. In the classical limit, the evolution of the mean value $\langle a\rangle_{k}$ in time is described by the Einstein-Friedmann equations. The observed part of the present-day universe has the 'radius' $a \sim 10^{28} \mathrm{~cm}$, and its total mass can be estimated as $M \sim 10^{80} \mathrm{GeV}$. In dimensionless units it corresponds to the condition $a \sim M \sim 10^{61}$, which agrees with (18), if the mean value $\langle a\rangle_{k}$ is identified with the classical value of the scale factor $a$.

Under the expansion in accordance with the Hubble law, $(d / d t) a(t)=H(t) a(t)$, the mass of a condensate in the universe changes as follows ${ }^{3}$

$$
\frac{d M_{k}(t)}{d t}=H(t) M_{k}(t)
$$

Since $\phi$-particles are indistinguishable and their masses do not change with time, the same equation holds for the number of undecayed particles,

$$
\frac{d \delta N_{\phi}(t)}{d t}=H(t) \delta N_{\phi}(t) .
$$

\footnotetext{
${ }^{2}$ In units under consideration, the value $\frac{1}{2} a^{3}$ is the dimensionless comoving volume of the universe, while $\mathrm{H}^{2}$ corresponds to the dimensionless critical density.

${ }^{3}$ In the standard cosmological model with the cosmological constant, the similar relation takes place. During the expansion the vacuum energy density remains constant $\rho_{\Lambda}=$ const, but the energy $M_{\Lambda}=\frac{1}{2} a^{3} \rho_{\Lambda}$ evolves as $\frac{d M_{\Lambda}}{d t}=3 H M_{\Lambda}$.
}

We suppose that $\phi$-particle, neutron, and proton decay independently with some decay rates $\Gamma_{\phi}=\Gamma_{\bar{\phi}}$, $\Gamma_{n}=\Gamma_{\bar{n}}$, and $\Gamma_{p}=\Gamma_{\bar{p}}$. The $\phi$-particles are supposed to be unstable ones. Then, taking into account twochannel $\phi$-particle decay as in the schemes (1) and (2), we can write the equations of the evolution of amounts of unstable particles in the form

$$
\begin{array}{r}
\frac{d \delta N_{\phi}(t)}{d t}=-\lambda(t) \delta N_{\phi}(t), \\
\frac{d \delta N_{n}(t)}{d t}=-\Gamma_{n}(t) \delta N_{n}(t)+\Gamma_{\phi}(t) \delta N_{\phi}(t), \\
\frac{d \delta N_{p}(t)}{d t}=-\Gamma_{p}(t) \delta N_{p}(t)+\Gamma_{n}(t) \delta N_{n}(t),
\end{array}
$$

where

$$
\lambda(t)=\Gamma_{\phi}(t)-H(t)
$$

is an effective decay rate which takes into account the decay of $\phi$-particles and the increase in the mass of a condensate during the expansion of the universe. According to (21), the number of neutrons (protons) decreases as a result of their decay and increases due to the decay of $\phi$-particles (neutrons). Since $\Gamma_{p} \ll \Gamma_{n}$, one can take $\Gamma_{p}=\Gamma_{\bar{p}}=0$. Choosing the initial conditions as follows

$$
\delta N_{\phi}\left(t^{\prime}\right)=N, \quad \delta N_{n}\left(t^{\prime}\right)=0, \quad \delta N_{p}\left(t^{\prime}\right)=0,
$$

where $N$ is the number of $\phi$-particles at initial instant of time $t^{\prime}$ prior to emergence of protons and neutrons in the system ${ }^{4}$, we find the solution of the set (21)

$$
\begin{gathered}
\frac{\delta N_{\phi}(t)}{N}=e^{-\bar{\lambda} \Delta t} \\
\frac{\delta N_{n}(t)}{N}=\int_{t^{\prime}}^{t} d t_{1} \Gamma_{\phi}\left(t_{1}\right) e^{-\int_{t^{\prime}}^{t_{1}} d t_{2} \lambda\left(t_{2}\right)} e^{-\int_{t_{1}}^{t} d t_{2} \Gamma_{n}\left(t_{2}\right)} \\
\frac{\delta N_{p}(t)}{N}=\int_{t^{\prime}}^{t} d t_{1} \Gamma_{n}\left(t_{1}\right) \\
\times \int_{t^{\prime}}^{t_{1}} d t_{2} \Gamma_{\phi}\left(t_{2}\right) e^{-\int_{t^{\prime}}^{t_{2}} d t_{3} \lambda\left(t_{3}\right)} e^{-\int_{t_{2}}^{t_{1}} d t_{3} \Gamma_{n}\left(t_{3}\right)}
\end{gathered}
$$

where $\bar{\lambda}=\bar{\Gamma}_{\phi}-\bar{H}$ and

$$
\bar{\Gamma}_{\phi}=\frac{1}{\Delta t} \int_{t^{\prime}}^{t} d t_{1} \Gamma_{\phi}\left(t_{1}\right), \quad \bar{H}=\frac{1}{\Delta t} \int_{t^{\prime}}^{t} d t_{1} H\left(t_{1}\right)
$$

are the mean decay rate of $\phi$-particles and the mean Hubble expansion rate on the time interval $\Delta t=t-t^{\prime}$.

The decay rate of $\phi$-particles $\Gamma_{\phi}(t)$ is unknown. We assume that the decay rates $\Gamma_{\phi}$ and $\Gamma_{n}$ depend very weakly on averaging interval $\Delta t$. Then from (25) and (26) we obtain

$$
\begin{array}{r}
\frac{\delta N_{n}(t)}{N}=\frac{\bar{\Gamma}_{\phi}}{\bar{\Gamma}_{n}-\bar{\lambda}}\left(e^{-\bar{\lambda} \Delta t}-e^{-\bar{\Gamma}_{n} \Delta t}\right), \\
\frac{\delta N_{p}(t)}{N}=\frac{\bar{\Gamma}_{\phi}}{\bar{\lambda}} \\
\times\left[1+\frac{1}{\bar{\Gamma}_{n}-\bar{\lambda}}\left(\bar{\lambda} e^{-\bar{\Gamma}_{n} \Delta t}-\bar{\Gamma}_{n} e^{-\bar{\lambda} \Delta t}\right)\right],
\end{array}
$$

\footnotetext{
${ }^{4}$ For our universe $t^{\prime} \sim 10^{-6} \mathrm{~s}$.
} 
where $\bar{\Gamma}_{n}$ is the mean decay rate of neutron.

The equations (24), (28) and (29) are consistent with the conservation law of particles,

$$
\begin{aligned}
& \delta N_{\phi}(t)+\delta N_{n}(t)+\delta N_{p}(t) \\
& =N+\frac{\bar{H}}{\bar{\lambda}} N\left(1-e^{-\bar{\lambda} \Delta t}\right),
\end{aligned}
$$

where the second summand on the right-hand side takes into account the number of $\phi$-particles which have appeared in the universe under its expansion and then decayed.

In our universe today $\bar{\Gamma}_{n}=1.12 \times 10^{-3} \mathrm{~s}^{-1}, H_{0}=$ $69.7 \mathrm{~km} \mathrm{~s}^{-1} \mathrm{Mpc}^{-1}$ and the age $t_{0}=13.75 \mathrm{Gyr}$ [1]. Taking $\Delta t=t_{0}$ for estimation we find that

$$
H_{0} \Delta t=0.98, \quad \bar{\Gamma}_{n} \Delta t=4.86 \times 10^{14} .
$$

We have supposed that the decay of $\phi$-particle is caused by the action of the after-GUT interaction with the mass scale $M_{X}$. Then the inequality $\bar{\Gamma}_{\phi} \ll \bar{\Gamma}_{n}$ must hold, and

$$
\bar{\lambda} \ll \bar{\Gamma}_{n}
$$

Under this condition, the number of baryons $\delta N_{p}$ in the expanding universe obeys the law

$$
\frac{\delta N_{p}(t)}{N}=\frac{\bar{\Gamma}_{\phi}}{\bar{\lambda}}\left[1-e^{-\bar{\lambda} \Delta t}\right]
$$

The ratio $\frac{\Omega_{B}}{\Omega_{D E}}$ can be written as follows

$$
\frac{\Omega_{B}}{\Omega_{D E}}=\sqrt{\frac{g_{p}}{g_{\phi}}} \frac{\bar{\Gamma}_{\phi}}{\bar{\lambda}}\left[e^{\bar{\lambda} \Delta t}-1\right],
$$

where $g_{p}=G m_{p}^{2}=0.59 \times 10^{-38}$ and $g_{\phi}=G m_{\phi}^{2}$ are the dimensionless gravitational coupling constants for proton and $\phi$-particle, respectively, $G=6.707 \times 10^{-39}$ $\mathrm{GeV}^{-2}$ is the Newtonian gravitational constant.

If $\bar{\Gamma}_{\phi} \gg \bar{H}$, then $\bar{\lambda} \approx \bar{\Gamma}_{\phi}$ and

$$
\frac{\Omega_{B}}{\Omega_{D E}}=\sqrt{\frac{g_{p}}{g_{\phi}}}\left[e^{\bar{\Gamma}_{\phi} \Delta t}-1\right] .
$$

This limit was considered in [18].

If $\bar{\Gamma}_{\phi} \ll \bar{H}$, then $\bar{\lambda} \approx-\bar{H}$ and

$$
\frac{\Omega_{B}}{\Omega_{D E}}=0
$$

It means that in this case baryons (ordinary matter) are not produced in the universe.

If $\bar{\Gamma}_{\phi} \approx \bar{H}$, and $\bar{\lambda} \Delta t \ll 1$, then

$$
\frac{\Omega_{B}}{\Omega_{D E}}=\sqrt{\frac{g_{p}}{g_{\phi}}} \bar{\Gamma}_{\phi} \Delta t\left[1+\frac{1}{2} \bar{\lambda} \Delta t+\ldots\right] .
$$

The ratio (37) describes the case, when the number of $\phi$-particles remains almost unchanged during the expansion of the universe, $\delta N_{\phi}(t) \approx N=$ const. It means that the amount of a condensate in the universe is semipermanent, but its energy density diminishes during the expansion. Then the coupling constant $g_{\phi}$ is equal to

$$
g_{\phi}=g_{p}\left(\frac{\Omega_{D E}}{\Omega_{B}} \bar{H} \Delta t\right)_{\bar{\lambda}=0}^{2} .
$$

Using the WMAP and other data [1], $\Omega_{B}=0.046$, $\Omega_{D E}=0.718$, and (31), and setting $\bar{H} \Delta t \approx H_{0} \Delta t$, we obtain

$$
g_{\phi} \approx 254 g_{p}
$$

Under this assumption, the decay rate of $\phi$-particle is very close to the Hubble expansion rate $H_{0}$ and it equals to

$$
\bar{\Gamma}_{\phi} \approx 2.3 \times 10^{-18} \mathrm{~s}^{-1}
$$

This value is close to the value found in [18], where (20) was not taken into account. That case is equivalent to the limit $\bar{\Gamma}_{\phi} \gg \bar{H}$. Therefore the values (39) and (40) may be considered as realistic, since they have changed very slightly and seem almost model-independent.

The coupling constant (39) corresponds to the mass

$$
m_{\phi} \approx 16 m_{p}
$$

Taking into account that, according to the decay schema (1), the number of dark matter $\chi$-particles is almost equal to the number of baryons,

$$
\delta N_{\chi}(t) \approx \delta N_{p}(t)
$$

from (16) we find that

$$
\frac{\Omega_{B}}{m_{p}} \approx \frac{\Omega_{D M}}{m_{\chi}} .
$$

For the observed values of $\Omega_{B}$ and $\Omega_{D M}=0.227[1]$ it follows that

$$
\frac{\Omega_{D M}}{\Omega_{B}} \approx 5 .
$$

With the regard for the errors of measurement of $\Omega_{D M}$ and $\Omega_{B}$, one can take the value of the mass of $\chi$-particle equal to

$$
m_{\chi} \approx 5 m_{p}
$$

This value is in the range $m_{\chi} \sim 1-10 \mathrm{GeV}$ indicated in [14]. It agrees with the observations of CoGeNT [19], DAMA [20], and CDMS [21]. The equation (42) is a relationship between the dark matter and baryon chemical potentials with the precise value $c_{1}=1$ of the coefficient $c_{1}$ introduced in ADM models (cf. [14]).

The values of masses (41) and (45) show that the decay (1) occurs with the release of energy $Q \approx 10 m_{p}$ in the form of kinetic energy of decay products.

From the equations (16) it follows

$$
\frac{\Omega_{D E}}{\Omega_{D M}}=\frac{m_{\phi} \delta N_{\phi}}{m_{\chi} \delta N_{\chi}} .
$$

Using the observed values of $\Omega_{D E}$ and $\Omega_{D M}$, and the obtained values of the masses $m_{\phi}$ (41) and $m_{\chi}$ (45), we find that

$$
\delta N_{\phi} \approx \delta N_{\chi}
$$

It means that the chemical potentials of dark energy and dark matter coincide. The equation (47) is a manifestation of the so-called coincidence problem, $\frac{\Omega_{D E}}{\Omega_{D M}} \approx 3.2$.

In conclusion to this Section, we note that the low mass dark matter problem can be analyzed, for instance, within the context of the standard model with scalar dark matter, ADM models, or the minimal supersymmetric standard model with neutralino dark matter (see, e.g., the bibliographies in $[14,19,22,23,24])$. A different 
model based on the quantum cosmological approach involving the available data on the abundances of baryons and dark matter in our universe was proposed in [18]. Obtained restriction on the mass of dark matter particle $m_{\chi}<15 \mathrm{GeV}$, with the preference for $m_{\chi} \sim 5-10$ $\mathrm{GeV}$, agrees with the values given in $[14,19]$ and in this paper.

\section{Estimations of Coupling Con- stant and Mass Scale}

From (11) and (40), for $Q \approx 10 m_{p}$, we obtain

$$
M_{X} \approx \sqrt{\alpha_{X}} 0.5 \times 10^{12} m_{p}
$$

The same formula follows from (13) and (14). The coupling constant $\alpha_{X}$ must satisfy the inequality

$$
\alpha_{W}^{2} \sim 10^{-5}<\alpha_{X}^{2}<\alpha_{G}^{2} \sim 10^{-3} .
$$

It gives the value for the mass scale $M_{X}$ which lies in the interval

$$
0.3<\left(M_{X} \times 10^{-11} m_{p}^{-1}\right)<0.9
$$

and we can accept the following numerical values

$$
\alpha_{X} \sim \frac{1}{70} \quad \text { at } \quad M_{X} \sim 6 \times 10^{10} \mathrm{GeV}
$$

for the parameters of after-GUT interaction. The radius of action of the after-GUT force is

$$
R_{X}=M_{X}^{-1} \sim 3 \times 10^{-25} \mathrm{~cm} .
$$

It gives the following value

$$
n_{\phi} \sim M_{X}^{3} \sim 10^{73} \mathrm{~cm}^{-3}
$$

for the density of the dark energy quasiparticles considered as $\phi$-particles surrounded by virtual $X$-particle cloud. The $\phi$-particle is massive and it can, in principle, exhibit itself through the gravitational action, but its gravitational coupling constant $g_{\phi} \approx 1.5 \times 10^{-36}$ is very small. The after-GUT coupling $\alpha_{X} \sim 10^{-2}$ has the same order of magnitude as the fine structure constant $\alpha \approx \frac{1}{137}$. This allows to consider the subtle processes which occur via virtual $X$-particle exchange as more realistic for identification of dark matter and dark energy.

Let us consider the reaction

$$
\bar{\nu}+\phi \rightarrow \chi+n,
$$

in which dark matter particles are produced. In Fig. 2 the diagram of this reaction is shown.

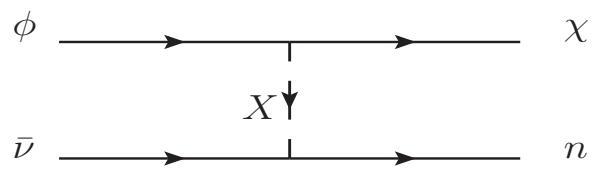

Figure 2. The diagram which describes the reaction (54).

The corresponding cross-section is equal to

$$
\sigma(\bar{\nu} \phi) \simeq \frac{\Gamma_{\phi}}{v M_{X}^{3}}
$$

where $v$ is the relative $\bar{\nu}-\phi$ motion velocity and the denominator is the incident flow of antineutrinos in the rest frame of $\phi$-particle. Since in this reference frame the velocity $v$ is equal to the velocity of the incident antineutrino, we can take $v \approx c$, and for the decay rate (40) and the mass scale $M_{X}$ from (51) we obtain

$$
\sigma(\bar{\nu} \phi) \sim 0.7 \times 10^{-74} \mathrm{GeV}^{-2} .
$$

Dark matter particles can be produced in another reaction as well,

$$
\bar{n}+\phi \rightarrow \chi+\nu
$$

For nonrelativistic antineutrons the cross-section of this process is

$$
\sigma(\bar{n} \phi) \simeq \frac{0.7}{\sqrt{E_{\bar{n}}}} \frac{\Gamma_{\phi}}{M_{X}^{3}},
$$

where $E_{\bar{n}}$ is the kinetic energy of antineutron taken in $\mathrm{GeV}$. For thermal and fast antineutrons the crosssections are equal to

$$
\begin{gathered}
\sigma(\bar{n} \phi) \sim 10^{-69} \mathrm{GeV}^{-2} \text { for } E_{\bar{n}}=0.25 \times 10^{-10} \mathrm{GeV} \\
\sigma(\bar{n} \phi) \sim 1.6 \times 10^{-73} \mathrm{GeV}^{-2} \text { for } E_{\bar{n}}=10^{-3} \mathrm{GeV}
\end{gathered}
$$

respectively. These values are much less than an elastic scattering cross-section of dark matter particle with nucleus $\sigma \sim 10^{-13} \mathrm{GeV}^{-2}$ used for the estimation of a mass of dark matter particle $[14,19]$.

\section{Conclusion Remarks}

Thus, one can conclude that the observed values of the densities $\Omega_{B}, \Omega_{D M}$, and $\Omega_{D E}$ in the model of the decays (1) and (2) lead to the values of mass of dark energy particle $\sim 15 \mathrm{GeV}$ and mass of dark matter particle $\sim 5 \mathrm{GeV}$. The obtained masses of dark energy and dark matter particles are consistent with the parameters of after-GUT interaction (51) and the decay rate $\Gamma_{\phi}(40)$. The parameters of after-GUT interaction are in so-called gauge desert - the domain between the electroweak and grand unification scales.

The density of the dark energy particles (53) shows that, after space-averaging, the volume $\sim(2 \mathrm{~m})^{3}$ contains the same number of dark energy particles as the number of equivalent baryons $\sim 10^{80}$ in the observed part of our universe. The cross-sections of the reactions (54) and (57) via virtual $X$-particle exchange are very small, but they are finite.

\section{REFERENCES}

[1] C. Bennett, et al., arXiv:1212.5225 [astro-ph.CO]; G. Hinshaw, et al., arXiv:1212.5226 [astro-ph.CO].

[2] S.M. Cole et al. MNRAS 326, 255 (2001).

[3] M. Persic, P. Salucci, MNRAS 258, 14P (1992); P. Salucci, M. Persic, MNRAS 309, 923 (1999).

[4] L. Amendola, S. Tsujikawa, Dark Energy: Theory and Observations, Cambridge University Press, 2010.

[5] S. Weinberg, Rev. Mod. Phys. 61, 1 (1989). 
[6] P.J.E. Peebles, B. Ratra, Rev. Mod. Phys. 75, 559 (2003).

[7] I. Dymnikova, M.Yu. Khlopov, Mod. Phys. Lett. A 15, 2305 (2000).

[8] I. Dymnikova, M.Yu. Khlopov, Eur. Phys. J. C 20, 139 (2001).

[9] V.E. Kuzmichev, V.V. Kuzmichev, Acta Phys. Pol. B 39, 2003 (2008) [arXiv:0712.0465 [gr-qc]]; Acta Phys. Pol. B 39, 979 (2008) [arXiv:0712.0464 [gr-qc]]; Acta Phys. Pol. B 40, 2877 (2009) [arXiv:0905.4142 [gr-qc]]; Ukr. Phys. J. 55, 626 (2010).

[10] P.A.M. Dirac, Lectures on Quantum Mechanics, Belfer Graduate School of Science, Yeshiva University, 1964; Can. J. Math. 2, 129 (1950); Can. J. Math. 3, 1 (1951).

[11] B.S. DeWitt, Phys. Rev. 160, 1113 (1967).

[12] J.D. Brown, D. Marolf, Phys. Rev. D 53, 1835 (1996).

[13] E.W. Kolb, M.S. Turner, The Early Universe, AddisonWesley Publishing Company, 1990.

[14] A.L. Fitzpatrick, D. Hooper, K.M. Zurek, arXiv:1003.0014 [hep-ph].

[15] J. Beringer et al. (Particle Data Group), Phys. Rev. D 86, 010001 (2012).
[16] R.H.Dicke, in: Gravitation and Relativity, eds. HongYee Chiu, W.F. Hoffmann, Benjamin, 1964.

[17] V.E. Kuzmichev, V.V. Kuzmichev, Ukr. Phys. J. 50, 321 (2005) [arXiv:astro-ph/0510763].

[18] V.E. Kuzmichev, V.V. Kuzmichev, in: Trends in Dark Matter Research, ed. J.V. Blain, Nova Science, 2005 [astro-ph/0405455]; Ukr. J. Phys. 48, 801 (2003) [arXiv:astro-ph/0301017]]; arXiv:astroph/0302173 (2003); in: Selected Topics in Theoretical Physics and Astrophysics, eds. A.K. Motovilov, F.M.Pen'kov, JINR, Dubna, 2003.

[19] C.E. Aalseth et al. (CoGeNT), Phys. Rev. Lett. 106, 131301 (2011) [arXiv:1002.4703 [astro-ph.CO]].

[20] R. Bernabei et al. (DAMA), Eur. Phys. J. C 56, 333 (2008).

[21] Z. Ahmed et al. (CDMS), arXiv:0912.3592 [astroph.CO].

[22] S.Andreas et al., arXiv:1003.2595 [hep-ph].

[23] D. Feldman, Z. Liu, P. Nath, arXiv:1003.0437 [hep-ph].

[24] A. Bottino, F. Donato, N. Fornengo, S. Scopel, Phys. Rev. D 81, 107302 (2010) [arXiv:0912.4025 [hep-ph]]; Phys. Rev. D 78, 083520 (2008). 